\title{
Stabilization of the Scanning Pattern for Three-Dimensional Phase-Sensitive OCT Modalities: Angiography, Relaxography, and Monitoring of Slow Processes
}

DOI: $10.17691 / \mathrm{stm} 2019.11 .2 .04$

Received March 25, 2019

P.A. Shilyagin, PhD, Senior Researcher, Laboratory of Highly Sensitive Optical Measurements';

L.A. Matveev, PhD, Senior Researcher, Laboratory of Wave Methods

for Studying Structurally Inhomogeneous Media';

E.B. Kiseleva, PhD, Senior Researcher, Scientific Laboratory of Optical Coherence Tomography, Institute of Experimental Oncology and Biomedical Technologies;

A.A. Moiseev, PhD, Senior Researcher, Laboratory of Highly Sensitive Optical Measurements ${ }^{1}$;

V.Y. Zaitsev, DSc, Head of the Laboratory of Wave Methods for Studying Structurally Inhomogeneous Media1;

A.A. Sovietsky, Senior Research Assistant, Laboratory of Wave Methods

for Studying Structurally Inhomogeneous Media';

D.V. Shabanov, PhD, Senior Researcher, Laboratory of Highly Sensitive Optical Measurements";

V.M. Gelikonov, DSc, Head of the Department of Nano-Optics and Highly Sensitive Optical Measurements ${ }^{1}$;

K.S. Yashin, PhD, Junior Researcher, Scientific Laboratory of Optical Coherence Tomography,

Institute of Experimental Oncology and Biomedical Technologies²; Neurosurgeon, University Clinic;

K.A. Achkasova, Student';

N.D. Gladkova, MD, DSc, Professor, Head of the Scientific Laboratory of Optical Coherence Tomography,

Institute of Experimental Oncology and Biomedical Technologies2;

G.V. Gelikonov, DSc, Head of the Laboratory of Highly Sensitive Optical Measurements ${ }^{1}$

${ }^{1}$ Federal Research Center Institute of Applied Physics, Russian Academy of Sciences, 46 Ulyanova St., Nizhny Novgorod, 603950, Russia;

2Privolzhsky Research Medical University, 10/1 Minin and Pozharsky Square, Nizhny Novgorod, 603005, Russia

New modalities of optical coherence tomography (OCT) are based not only on the analysis of light beam scattering by biological tissues, but also, to a greater extent, on the assessment of spatio-temporal changes in the speckle structure (both the amplitude and the speckle phase).

The aim of the study was to develop a method for eliminating parasitic vibrations in the scanning system, which interfere with the new OCT modalities such as angiography, relaxography, and slow process monitoring.

Materials and Methods. The task of stabilizing the beam scan trajectory was successfully solved by actively filtering the controlling voltage.

Results. The effect of the proposed approach application is demonstrated both on a phantom sample and examples of implementation of several modalities applied to real tissues, including: exudate visualization in vivo; OCT-angiography for visualization of microcirculation of the brain; OCT-relaxography on the example of mapping the time of mechanical relaxation of cartilage tissue; increasing the lateral resolution of OCT by compensating for defocusing.

Conclusion. Vibrations of the scanning system cause artifact speckle variations that mask the informative variations of the speckles due to the relative change of the position of the scatterers inside the biological tissue caused by Brownian motion, flow, and deformation. Without proper stabilization of the scanning pattern, it is impossible to implement these modalities with quality suitable for practical use.

Key words: scan pattern; optical coherence tomography; OCT; OCT-angiography, OCT-relaxography; monitoring of slow processes.

\section{Introduction}

Optical coherence tomography (OCT) is a rapidly developing, minimally invasive method for visualization of biological tissues that does not require contrast agents. The OCT has already taken a leading position among the optical diagnostic methods due to its ability to rapidly obtain and process the images; in addition, OCT features the well-known polarization sensitivity, such rapidly developing modalities as angiography and elastography, as well as novel functions, such as lymphoangiography, relaxography, and monitoring

Corresponding author: Elena B. Kiseleva, e-mail: kiseleva84@gmail.com 
of slow processes [1, 2]. OCT devices produce tissue structure images with a resolution up to a few micrometers to a depth of $1.5 \mathrm{~mm}$. The main areas of OCT application are ophthalmology [3] and interventional cardiology $[4,5]$. OCT is widely used in dermatology, which is partly due to the convenient accessibility of skin as an object of the study $[6,7]$.

Currently, much attention of researches is paid to studying possibilities of OCT in oncology for diagnostic tumor assessment $[8,9]$, determining tumor boundaries [10], and evaluating the efficacy of antitumor treatment [11].

A major element of the OCT instruments is the scanning system, which controls the movement of the probe beam along the surface under study. A series of A-scans recorded synchronously with this movement (coordinate $X$ ) is called the B-scan. Three-dimensional OCT images are formed from a set of B-scans recorded with a sequential shift along the coordinate orthogonal to the scanning direction in one B-scan (coordinate $Y$ ). For a fixed number $(A)$ of $A$-scans in one B-scan, the scanning speed along the $X$ coordinate is $A$ times higher than the scanning speed along the $Y$ coordinate, which makes it possible to use the terms "fast" and "slow" scan coordinates.

The array of beam trajectories is called "the scanning pattern". The most common is a raster scanning (Figure 1 (a), (b)) [12], in which $X$ and $Y$ are the Cartesian rectangular coordinates although other scanning bases are also used. For example, in some studies of the macula region and the optic nerve disk in the human retina, the B-scan looks like a multi-pointed star (Figure 1 (c)) [13]; in studies of the optic nerve, a circle shaped scanning is often used (Figure 1 (d)) $[12,14]$. When using resonant endoscopic forwardlooking probes [15-17], the B-scan shape is defined by Lissajous figures (Figure 1 (e)). In this case, the "slow" coordinate of the scan is determined by the phase difference between the harmonics of the signal that controls the optical fiber motion in the orthogonal directions. In studies on the inner walls of blood vessels, esophagus or respiratory tract, the B-scan is formed by the rotation of the probe around its axis [18]. In that case, the azimuth coordinate serves the "fast" one, and the "slow" coordinate corresponds to the longitudinal displacement of the probe along its axis (Figure 1 (f)).

To implement multimodal OCT options in systems developed at the Institute of Applied Physics, Russian Academy of Sciences (Nizhny Novgorod), raster scanning is used to ensure the continuity of the information; here, the beam movement along the $X$ coordinate proceeds in the opposite directions for the odd and even B-scans, intermittently. This type of scanning makes it possible to implement the polarization-sensitive (PS) modality based on a double passage of the probe beam in the same plane; in this case, the polarization of the reference wave changes for the orthogonal one on each pass [19]. Due to the bio-tissue specificity, the diagnostically significant transverse sizes of biological structures, recorded with the regular or PS modalities of OCT, significantly exceed the uncertainty of the scanning beam position caused by mechanical resonances of the scanning element. In view of this, the issue of high-grade scanning pattern was not addressed until the invention of phase-sensitive OCT modalities.

However, for implementation of signal-sensitive modalities of OCT (such as angiography, mapping of tissue deformations and monitoring of Brownian motion), it is necessary to enhance the stabilization of the scanning system to ensure the reproducibility of the beam passage without deviating from the previous path. For example, in angiography and elastography, at each point of each B-scan pair, the phase difference of the resulting OCT signal is calculated $[20,21]$ and then used for the visualization and estimation of displacements of sub-resolution scatterers. However, if the scanning

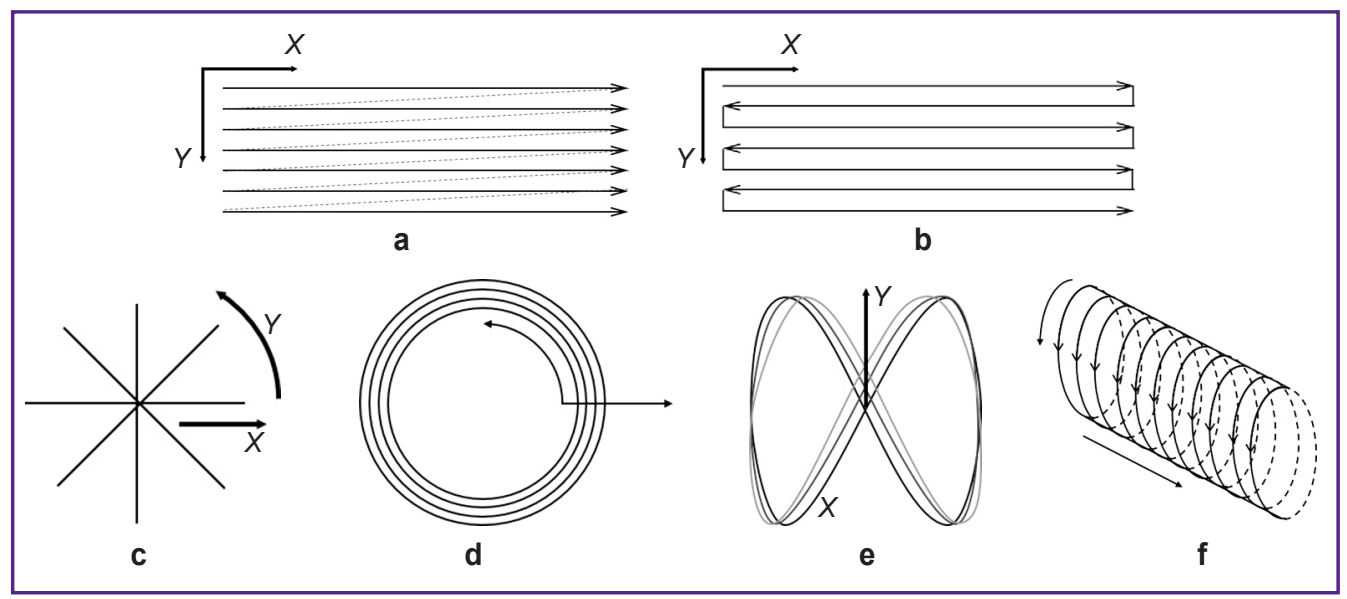

Figure 1. The most typical OCT scan patterns:

(a) raster with breaks; (b) raster without breaks; (c) star-shaped; (d) concentric; (e) Lissajous figures; (f) cylindrical 
system does not ensure sufficient reproducibility of the beam trajectories, this can lead to erroneous estimates of not only phase variations, but even speckle-amplitude variations that are less sensitive to motions of scatterers.

\section{Materials and Methods}

This paper presents an approach developed in our group to stabilize the trajectory of continuous threedimensional raster OCT scanning. The implementation of the proposed method is exemplified by a number of clinical and preclinical applications:

1) visualization of exudative fluid in vivo (based on stabilized amplitude processing) in a patient with exudative otitis media;

2) visualization of blood microcirculation (based on stabilized phase-sensitive processing of B-scans) in the brains of healthy and tumor-implanted rats;

3) mapping of mechanical-relaxation rate (based phase processing of $3 \mathrm{D}$ arrays) in porcine rib cartilage after needle puncture;

4) improving the transverse resolution of OCT by compensating for divergence of a focused beam outside the focal plane (based on phase processing) with demonstration on a phantom.

\section{Results}

Stabilization of the continuous raster $3 D$ scanning in OCT. A key point in the implementation of continuous raster $3 \mathrm{D}$ scanning is the suppression of excitation of higher resonance harmonics oscillations while tilting direction inversion of the scanning element. In Figure 2 (a), the structural en-face image of an OCT phantom (BioMimic, Canada) that represents a uniformly scattering medium is shown. Periodic distortions and discernable artefactual inhomogeneities are visible in the image although they are absent in the real structure of the object. Distortions are caused by scanning irregularity and may lead to erroneous interpretations of OCT images and reduce the diagnostic value of the method.
The phase components of OCT data which is not commonly used for imaging are even more sensitive to parasitic micro-displacements of the scanning beam component. The difference between phase components of continuously recorded scans of rigid medium with fixed scatterers is imaged in Figure 2 (b). The structurelike image is an artifact which presence on the figure and caused by unwanted oscillations of scanning mirror as a result of stimulation of higher harmonics of its mechanical resonance by "slow" axis controlling voltage steps.

To eliminate these artefacts, we have developed an approach based on the active filtering of the controlling voltage. For this purpose, we used both a filtering unit and a simultaneous fine pre-tuning of the controlling signal to minimize the influence of electric circuit nonlinearity. It allows avoiding stimulation of higher resonant harmonics caused by the shape of the discrete steps of the controlling voltage at the digital-to-analog unit output. The proposed system allowed us to get rid of the mirror tremor artifacts both in the traditional enface structural images (Figure 2 (c)) and in the dynamic structure analysis (Figure $2(\mathrm{~d})$ ).

Visualization of exudative fluid in vivo. After eliminating parasitic vibrations of scanning element, it becomes possible to visualize the fluid accumulating in the middle ear in patients with exudative otitis. In Figure 3 (a), a fragment of exudative fluid that reaches the temporal bone and the thinned eardrum is clearly visible. The fact that the refractive index of the exudative fluid $n$ is different from the unity causes the appearance of visible "breaks" in the underlying tissues (e.g. the temporal bone in Figure 3 (a)) under the interface between the fluid and the ambient air. In addition, the speckle structure of the exudate zone differs from that of the overlying and underlying areas. This is illustrated in Figure 3 (b), which reflects the section shown by the dash-dotted line in Figure 3 (a). The image in Figure 3 (b) was reconstructed from a set of 100 individual OCT images obtained from a single plane at different time points (the interval between individual B-scans was about $25 \mu \mathrm{s}$ ).
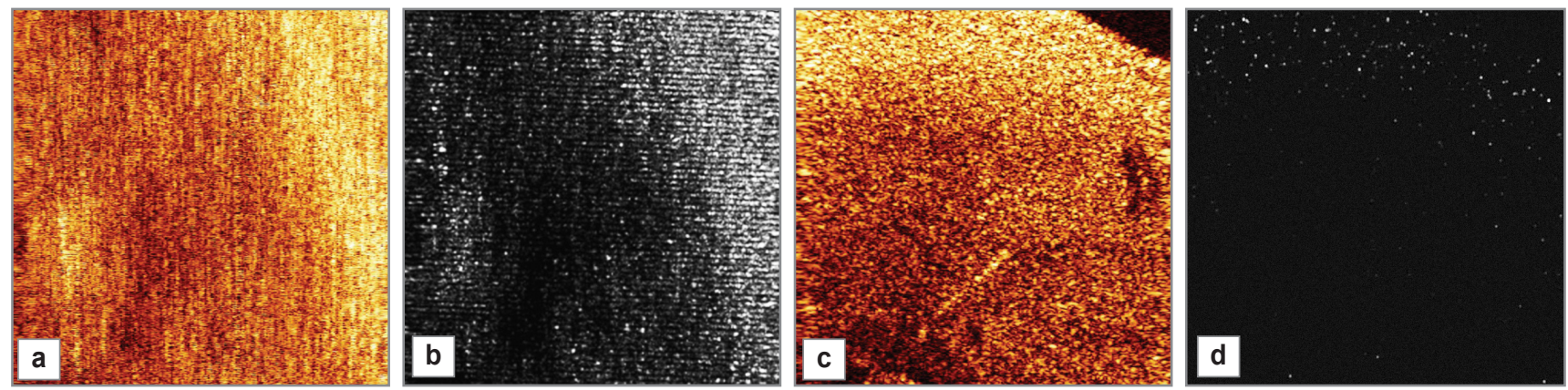

Figure 2. Images obtained upon excitation of the higher resonance harmonics in the scanning element:

(a) en-face image of the test medium upon excitation of the higher harmonics; (b) phase instability of the image (a); (c) en-face image of the test medium a corrected for excitation of the higher harmonics; (d) phase instability of the image (c) 

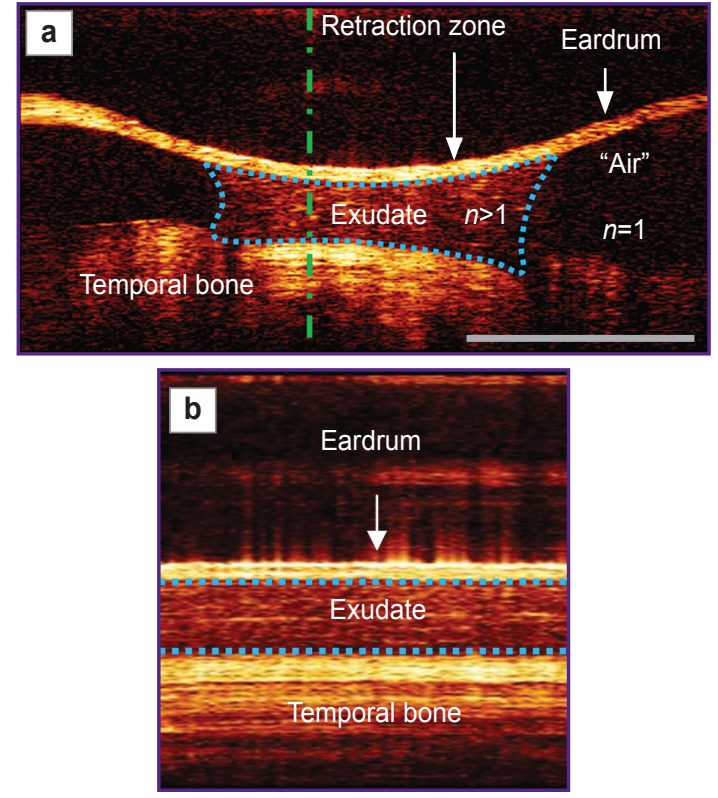

Figure 3. Visualization of exudative fluid in vivo:

(a) standard OCT image; (b) time-resolved image; the exudative fluid differs from the ambient air by the refractive index value, which is visualized as breaks in the underlying structure (temporal bone - (a)); this fluid differs from solid structures by the dynamic characteristics of the scatterers, which manifests in different effective lengths of the speckles along the horizontal coordinate (b); bar $-1 \mathrm{~mm}$

Due to the fact that individual scatterers in a fluid are movable, they are involved in the Brownian motion; as a result, the speckles in the "liquid" part of the image (exudate) have a shorter horizontal length compared to the speckles of the "hard" structure. Evaluation of speckle brightness and mobility can provide information about the viscosity of the exudate and help choose the treatment tactics (conservative or surgical) [22, 23]. However, a difference in the speckle lengths (their mobility) can be measured only if the scanning pattern is stable; then the inverse value of the image length would reflect the real mobility of the scatterers and not artificial vibrations during the scanning.
Thus, stabilization of the scanning trajectory and elimination of parasitic vibrations are crucial for performing the visualization of the exudate area, and are also helpful in assessing the exudate viscosity, which is based on the dynamic characteristics of the OCT image speckles.

OCT visualization of microcirculation of the rat brain, intact and containing a glial tumor. Today's methods of OCT-angiography are based on the analysis of local changes in the speckles [24]. In this approach, both the amplitude and the phase of the OCT signal are considered [25]. Therefore, stabilization of the scanning pattern is crucial for the quality of OCT-angiography.

In many experimental studies, OCT-angiography is used to assess the ischemic processes in the brain $[26,27]$. Studies on the blood vessel network in brains affected by gliomas are rare [28]. The present report demonstrates the potential of using OCT-angiography for monitoring changes in the brain microvasculature during the development of brain tumors.

OCT imaging of the rat brain microcirculation bed under normal condition and in the presence of implanted and growing experimental glioma C6 was performed after craniotomy with the help of an object lens and ultrasound gel [29].

Animal maintenance and experimentation were carried out in accordance with the Guide for the Care and Use of Laboratory Animals (National Research Council, 2011). The work with the animals was performed according to the ethical principles of the European Convention for the Protection of Vertebrate Animals used for Experimental and Other Scientific Purposes (Strasbourg, 2006) and approved by the Ethics Committee of the Privolzhsky Research Medical University.

Angiographic images were obtained in real time in parallel with acquiring of the 3D sets of OCT data (which took $26 \mathrm{~s}$ ) that represented a stack of $2 \mathrm{D}$ pictures of blood vessels located at various depths in the tissue (up to $1 \mathrm{~mm}$ ). These data were projected onto a plane perpendicular to the tissue surface (Figure 4) [30].

Identification and separation of the blood vessel images from the total OCT data were performed using
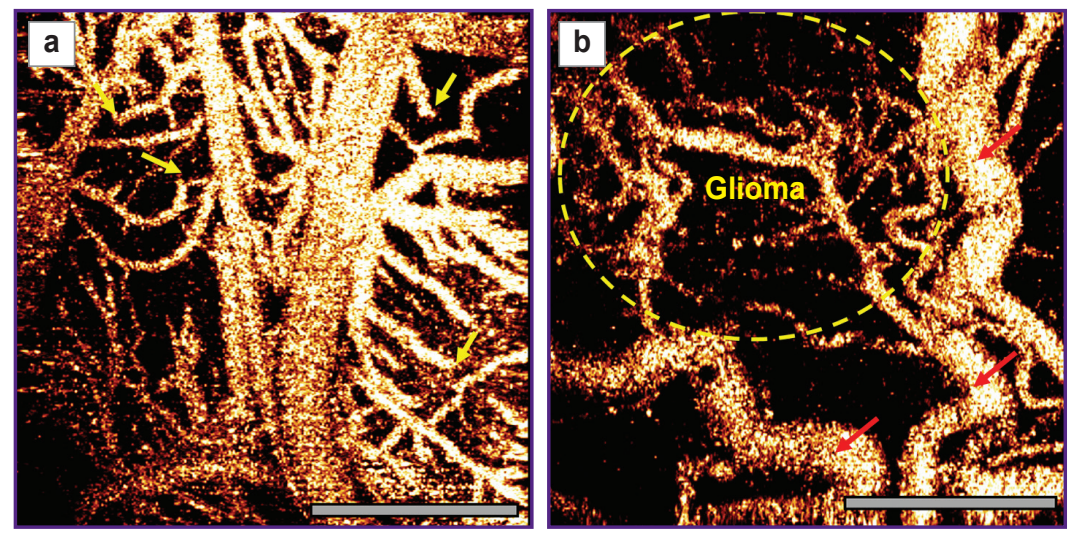

Figure 4. OCT visualization of brain microcirculation in intact and C6-glioma implanted rat:

(a) a blood vessel network in the normal rat brain; the yellow arrows indicate vessels receding ("diving") deep into the brain tissue; (b) a blood vessel network in the area of tumor growth in the cerebral cortex; the red arrows indicate pathologically dilated blood vessels in the perifocal zone of the glioma tumor; bar $-1 \mathrm{~mm}$ 
high-pass filtering. This setup allows one to visualize areas with the presence of blood flow (movement of red blood cells); areas with no blood flow are not visible. As a result, blood vessel images are formed as an en-face projection of the maximum intensity of the filtered OCT signal at each point, calculated over the entire depth of the A-scan. The minimum diameter of the vessels that can be seen at this resolution is $15 \mu \mathrm{m}$.

The blood vessel network in the central and perifocal zone of the tumor notably differed from those in the brain of rats from the reference group (see Figure 4). The architectonics of the rat cerebral vascular bed is usually visualized on OCT-angiographic images in the form of a network with a trunk type of branching. A central wellvisible vessel gives rise to second-order vessels that, in turn, branch off, and so on (Figure 4 (a)). The yellow arrows show thin, as if breaking off, vessels changing their directions and going into the depths of the cortex, where they become inaccessible by OCT.

The angiographic examination of the vascular network in a $\mathrm{C} 6$ tumor is strikingly different from the norm. The vessels are predominantly thin, sinuous, of approximately uniform diameter (Figure 4 (b)), the yellow dotted oval). Notable is the state of the vessels in the perifocal area: the local vascular network is depleted, i.e., represented by thickened blood vessels of irregular shape (in some spots, vessels are dilated to form lacunas), as shown by the red arrows.

These features, even with the visual assessment of OCT-angiographic images, make it possible to accurately determine the central and perifocal tumor zones, as well as to distinguish between these areas and the normal tissue. The typical characteristics of brain microcirculation normally include the main type of branching, the presence of vessels of different diameters, and the definite direction of the vessels. In contrast, in tumor the vessels have no definite directions; they are tortuous and located chaotically. In the perifocal zone, the vascular network has a lower density and tortuosity; also the diameters of individual vessels are significantly increased.

Thus, the proposed system with scanning pattern stabilization allows one to use OCT-angiography for non-invasive mapping of vascular networks both in normal and pathological (oncological) conditions. This modality provides information in real time and does not require additional labels or dyes. We have demonstrated the ability of this modified OCT-angiography to detect changes in brain microcirculation in vivo caused by the tumor growth, which provides the rationale for the intraoperative application of this method in the future.

Mapping of mechanical elastic relaxation of cartilage tissue. The achieved reproducibility of the scanning trajectories ensures the consistency of different OCT data arrays in the case of sequentially recorded 3D volumes. In this mode, the volumes of 512 and 32 A-scans are created via the "fast" and "slow" coordinates, respectively, with an area of $2 \times 1 \mathrm{~mm}^{2}$ in $1.5 \mathrm{~s}$. The consistency of scanning trajectories makes it possible to implement phase methods of 3D OCT-elastography and OCT-relaxography [31] based on the advanced phasesensitive methods of local strain estimation [32-35]. The number of sequentially scanned volumes is limited only by the memory of the controlling computer. For the OCTrelaxography, in order to monitor the discussed here processes of slow tissue deformation, 90 volumes are sufficient.

In Figure 5, a 3D OCT-relaxography scan of porcine cartilage tissue punctured with a needle is shown. The needle was rapidly removed from the tissue just before the scanning started. The 3D reconstructed OCT image (Figure 5 (a)) shows the homogeneous structure of this cartilage fragment. The point of puncture is indicated by the arrow. Figure 5 (b) shows a 3D OCT-relaxogram,

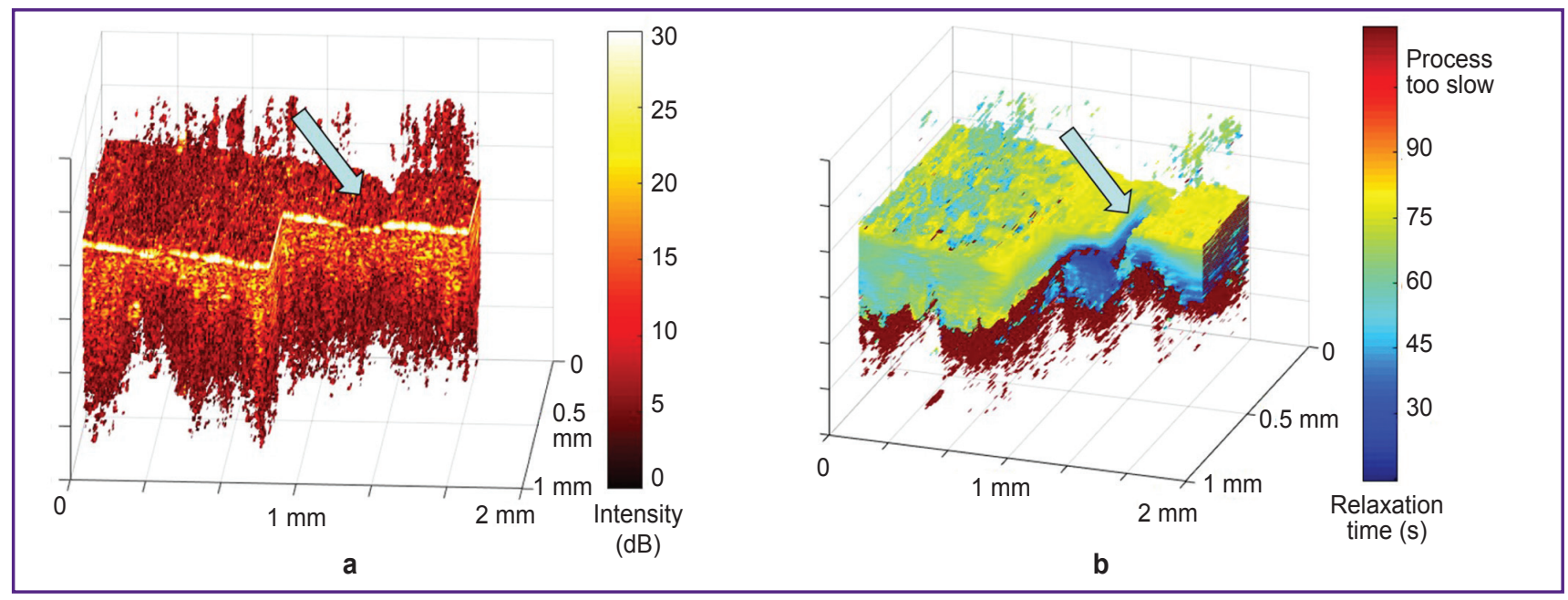

Figure 5. OCT visualization of mechanical relaxation in cartilage after removing the needle:

(a) structural 3D OCT image; (b) 3D OCT-relaxogram reflecting the spatial distribution of the relaxation time; blue color denotes the fast process, yellow and red - the slower ones; the needle withdrawal point is shown by the arrow 

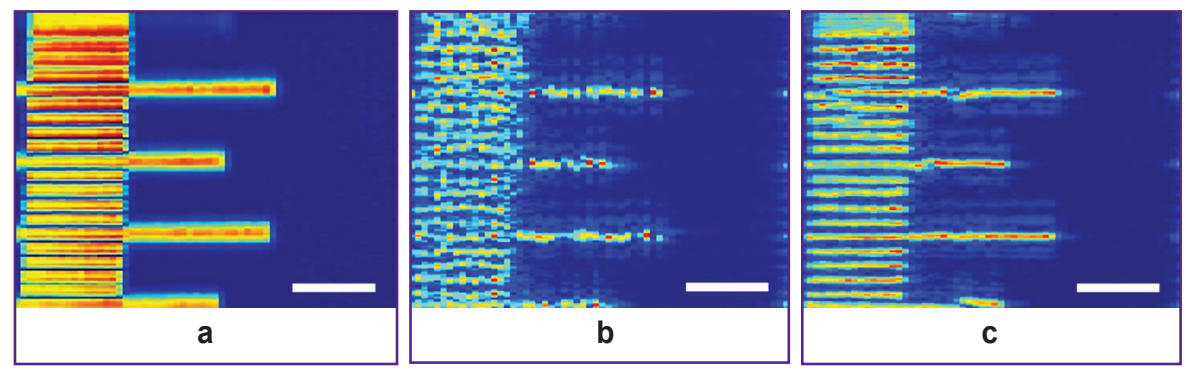

Figure 6. Example of the numerical refocusing of OCT images (a gauge):

(a) image taken outside the focal plane of the OCT-scanning beam; (b) numerical refocusing according to [38] with no scanning stabilization; (c) numerical refocusing of the data obtained with scanning stabilization; bar $-100 \mu \mathrm{m}$

where the relaxation time is displayed according to the color spectrum (on the right). The arrow indicates the puncture point. It is clearly seen that the relaxation time in the puncture area is noticeably lower (30-45 s) compared to the surrounding intact areas (60-80 s) (see Figure $5(b)$ ), which could not be detected with the initial structural OCT image. Note that the algorithm for calculating the relaxation time automatically discards most of the artifacts originated from the area above the sample surface.

Thus, using OCT-relaxography, slow deformations of cartilage tissues can be measured and analyzed. In the future, this approach can be used for monitoring internal changes in a cartilage during its drying, dehydration, saturation, or other processes.

Increasing the transverse resolution of OCT by compensating for the defocusing. In the conventional OCT, the transverse resolution is determined by the diffraction properties of the scanning beam. The usual requirement is to ensure the uniform resolution throughout the entire depth of the object. These requirements determine the standard size of the OCT transverse resolution, i.e., 15-20 $\mu \mathrm{m}$, approximately equal to the beam diameter in the focal plane. Significant decrease in this size reduces the length of the scanning beam's Rayleigh length and results in strong beam divergence outside the focal plane. This correspondingly strongly reduces the transverse resolution within the most part of the imaged depth except for the immediate vicinity of the focal plane. To solve the problem of obtaining higher resolution over the entire depth comparable to the resolution in the focal plane, the socalled numerical refocusing can be used [36-38].

The numerical refocusing for highly-focused OCT beams is possible, because the signal from each scatterer is measured from various directions within the scanning beam. These data can be coherently combined to obtain a final signal with an improved resolution. Since the numerical refocusing method is coherent and explicitly uses the full (with amplitude and phase) values of the scattered field (measured at different positions of the scanning beam), it is necessary to ensure the phase stability between two consecutive measurements.
Figure 6 shows OCT images of the test object (a gauge), obtained with a probe beam with a waist diameter of $7 \mu \mathrm{m}$ at a distance of three Rayleigh wavelengths from the focal plane.

In the first case (Figure $6(b)$ ), the measurements were carried out without ensuring phase stability; in the second case, phase stability was ensured at the stage of the data postprocessing (Figure 6 (c)). In the first case, an attempt of numerical compensation for defocusing failed since the loss of phase information between the consecutive measurements precluded any coherent operations with these data (see Figure 6 (b)). In the case of numerical refocusing under the condition of inter-measurement phase stabilization, it became possible to restore the resolution to the level equal to the resolution in the focal plane (see Figure 6 (c)).

Increasing the transverse resolution is an important step toward increasing the diagnostic value of OCT. Such an increase may open new venues for this modality, specifically in dermatology, where images with a resolution of $\sim 3 \mu \mathrm{m}$ may be of high diagnostic value and eventually replace skin biopsy procedures [39].

\section{Conclusion}

New modalities of OCT are based not only on the analysis of the averaged scattering amplitude of various tissue layers, but, to a greater degree, on the assessment of speckle structure variability (both amplitudes and phases of the speckles). To implement these modalities, one has to exclude parasitic vibrations in the scanning system, since such vibrations cause artefactual variations of the speckle structure. These variations mask the real variability of speckles caused by changes in the location of scattering elements in the biological tissue due to Brownian motion, flows and deformations.

In this study, the improved stability of the scanning trajectory was achieved by using active filtering of the controlling voltage. The successful use of the proposed approach is demonstrated on a phantom and real tissues by implementing several OCT modalities: in vivo visualization of an exudate; OCT-angiography for the visualization of brain microcirculation; improving the transverse resolution of OCT by compensating for the defocusing phenomenon; OCT-relaxography for mapping the mechanical relaxation time in a cartilage tissue.

Without applying the proposed method of stabilization of the scanning pattern, it is practically impossible to implement these modalities with an acceptable quality. 
Research funding. The development of the softand hard-ware for the correction of the resonance characteristics of the scanning element, model experiments, and visualization of exudative fluid in the tympanic cavity were carried out with the support of grant No.17-15-01507 from the Russian Science Foundation. The development and software implementation of scanning patterns for obtaining angiographic images of the brain in intact and glioma-implanted animals were supported by grant No.MK-6634.2018.7 from the Russian President grant for young scientists. The implementation of OCT-relaxography and the study of mechanical relaxation of cartilage tissue were supported by grant No.16-02-00642_a from the Russian Foundation for Basic Research. The development of approaches to improving the transverse resolution in OCT was supported by grant No.17-72-20249 from the Russian Science Foundation.

Conflict of interest. The authors have no conflicts of interest.

\section{References}

1. Drexler W., Liu M., Kumar A., Kamali T., Unterhuber A., Leitgeb R.A. Optical coherence tomography today: speed, contrast, and multimodality. J Biomed Opt 2014; 19(7): 071412, https://doi.org/10.1117/1.jbo.19.7.071412.

2. de Boer J., Leitgeb R., Wojtkowski M. Twenty-five years of optical coherence tomography: the paradigm shift in sensitivity and speed provided by Fourier domain OCT. Biomed Opt Express 2017; 8(7): 3248-3280, https://doi.org/10.1364/ boe.8.003248.

3. Goldman D., Waheed N.K., Duker J.S. Atlas of retinal OCT: optical coherence tomography. Elsevier; 2017; 224 p.

4. Kini A., Narula J., Vengrenyuk Y., Sharma S. Atlas of coronary intravascular optical coherence tomography. Springer International Publishing; 2018, https://doi.org/10.1007/978-3319-62666-6.

5. Dohad S., Zhu A., Krishnan S., Wang F., Wang S., Cox J., Henry T.D. Optical coherence tomography guided carotid artery stent procedure: technique and potential applications. Catheter Cardiovasc Interv 2018; 91(3): 521-530, https://doi.org/10.1002/ccd.27344.

6. Sattler E., Kästle R., Welzel J. Optical coherence tomography in dermatology. J Biomed Opt 2013; 18(6): 061224, https://doi.org/10.1117/1.jbo.18.6.061224.

7. Olsen J., Themstrup L., Jemec G.B. Optical coherence tomography in dermatology. G Ital Dermatol Venereol 2015; 150(5): 603-615.

8. Chin L., Latham B., Saunders C.M., Sampson D.D., Kennedy B.F. Simplifying the assessment of human breast cancer by mapping a micro-scale heterogeneity index in optical coherence elastography. J Biophotonics 2017; 10(5): 690-700, https://doi.org/10.1002/jbio.201600092.

9. Gubarkova E.V., Sovetsky A.A., Zaitsev V.Y., Matveyev A.L., Vorontsov D.A., Sirotkina M.A., Matveev L.A., Plekhanov A.A., Pavlova N.P., Kuznetsov S.S., Vorontsov A.Yu., Zagaynova E.V., Gladkova N.D. OCT-elastography-based optical biopsy for breast cancer delineation and express assessment of morphological/molecular subtypes. Biomed Opt Express 2019; 10(5): 2244-2263, https://doi.org/10.1364/boe.10.002244.
10. van Manen L., Dijkstra J., Boccara C., Benoit E., Vahrmeijer A.L., Gora M.J., Mieog J.SD. The clinical usefulness of optical coherence tomography during cancer interventions. J Cancer Res Clin Oncol 2018; 144(10): 1967-1990, https:// doi.org/10.1007/s00432-018-2690-9.

11. Sirotkina M.A., Matveev L.A., Shirmanova M.V., Zaitsev V.Y., Buyanova N.L., Elagin V.V., Gelikonov G.V., Kuznetsov S.S., Kiseleva E.B., Moiseev A.A., Gamayunov S.V., Zagaynova E.V., Feldchtein F.I., Vitkin A., Gladkova N.D. Photodynamic therapy monitoring with optical coherence angiography. Sci Rep 2017; 7(1): 41506, https://doi. org/10.1038/srep41506.

12. Chauhan B.C., Stevens K.T., Levesque J.M., Nuschke A.C., Sharpe G.P., O'Leary N., Archibald M.L., Wang $X$. Longitudinal in vivo imaging of retinal ganglion cells and retinal thickness changes following optic nerve injury in mice. PLoS One 2012; 7(6): e40352, https://doi.org/10.1371/ journal.pone.0040352.

13. Gupta A., Laxmi G., Nittala M.G., Raman R. Structural and functional correlates in color vision deficiency. Eye 2011; 25(7): 909-917, https://doi.org/10.1038/eye.2011.87.

14. Taibbi G., Peterson G.C., Syed M.F., Vizzeri G. Effect of motion artifacts and scan circle displacements on Cirrus HD-OCT retinal nerve fiber layer thickness measurements. Invest Ophthalmol Vis Sci 2014; 55(4): 2251-2258, https://doi. org/10.1167/iovs.13-13276.

15. Huo L., Xi J., Wu Y., Li X. Forward-viewing resonant fiber-optic scanning endoscope of appropriate scanning speed for 3D OCT imaging. Opt Express 2010; 18(14): 14375-14384, https://doi.org/10.1364/oe.18.014375.

16. Moon S., Lee S.W., Rubinstein M., Wong B.J., Chen Z. Semi-resonant operation of a fiber-cantilever piezotube scanner for stable optical coherence tomography endoscope imaging. Opt Express 2010; 18(20): 21183-21197, https://doi. org/10.1364/oe.18.021183.

17. Park H.-C., Seo Y.-H., Jeong K.-H. Lissajous fiber scanning for forward viewing optical endomicroscopy using asymmetric stiffness modulation. Opt Express 2014; 22(5): 5818-5825, https://doi.org/10.1364/oe.22.005818.

18. Bezerra H.G., Costa M.A., Guagliumi G., Rollins A.M., Simon D.I. Intracoronary optical coherence tomography: a comprehensive review clinical and research applications. JACC Cardiovasc Interv 2009; 2(11): 1035-1046, https://doi. org/10.1016/j.jcin.2009.06.019.

19. Gelikonov V.M., Romashov V.N., Shabanov D.V., Ksenofontov S.Y., Terpelov D.A., Shilyagin P.A., Gelikonov G.V., Vitkin I.A. Cross-polarization optical coherence tomography with active maintenance of the circular polarization of a sounding wave in a common path system. Radiophys Quantum El 2018; 60(11): 897-911, https://doi.org/10.1007/s11141-018-9856-9.

20. Matveyev A.L., Matveev L.A., Sovetsky A.A., Gelikonov G.V., Moiseev A.A., Zaitsev V.Y. Vector method for strain estimation in phase-sensitive optical coherence elastography. Laser Phys Lett 2018; 15(6): 065603, https://doi. org/10.1088/1612-202x/aab5e9.

21. Moiseev A., Ksenofontov S., Sirotkina M., Kiseleva E., Gorozhantseva M., Shakhova N., Matveev L., Zaitsev V., Matveyev A., Zagaynova E., Gelikonov V., Gladkova N., Vitkin A., Gelikonov G. Optical coherence tomography-based angiography device with real-time angiography B-scans visualization and hand-held probe for everyday clinical use. J Biophotonics 2018; 11(10): e201700292, https://doi. org/10.1002/jbio.201700292. 
22. Shilyagin P.A., Novozhilov A.A., Abubakirov T.E., Gelikonova V.G., Terpelov D.A., Matkivsky V.A., Gelikonov G.V., Shakhov A.V., Gelikonov V.M. Time domain optical coherence tomography is a useful tool for diagnosing otitis media with effusion. Laser Phys Lett 2018; 15(9): 096201, https://doi.org/10.1088/1612-202x/aacb4c.

23. Monroy G.L., Pande P., Shelton R.L., Nolan R.M., Spillman D.R. Jr., Porter R.G., Novak M.A., Boppart S.A. Noninvasive optical assessment of viscosity of middle ear effusions in otitis media. J Biophotonics 2017; 10(3): 394-403, https:// doi.org/10.1002/jbio.201500313.

24. Mariampillai A., Standish B.A., Moriyama E.H., Khurana M., Munce N.R., Leung M.K., Jiang J., Cable A., Wilson B.C., Vitkin I.A., Yang V.X. Speckle variance detection of microvasculature using swept-source optical coherence tomography. Opt Lett 2008; 33(13): 1530-1532, https://doi. org/10.1364/ol.33.001530.

25. Mahmud M.S., Cadotte D.W., Vuong B., Sun C., Luk T.W., Mariampillai A., Yang V.X. Review of speckle and phase variance optical coherence tomography to visualize microvascular networks. J Biomed Opt 2013; 18(5): 050901, https://doi.org/10.1117/1.jbo.18.5.050901.

26. Baran U., Wang R.K. Review of optical coherence tomography based angiography in neuroscience. Neurophotonics 2016; 3(1): 010902, https://doi.org/10.1117/1. nph.3.1.010902.

27. Choi W.J., Li Y., Qin W., Wang R.K. Cerebral capillary velocimetry based on temporal OCT speckle contrast. Biomed Opt Express 2016; 7(12): 4859-4873, https://doi.org/10.1364/ boe.7.004859.

28. Vakoc B.J., Lanning R.M., Tyrrell J.A., Padera T.P., Bartlett L.A., Stylianopoulos T., Munn L.L., Tearney G.J., Fukumura D., Jain R.K., Bouma B.E. Three-dimensional microscopy of the tumor microenvironment in vivo using optical frequency domain imaging. Nat Med 2009; 15(10): 1219-1223, https://doi.org/10.1038/nm.1971.

29. Yashin K.S., Kiseleva E.B., Gubarkova E.V., Matveev L.A., Karabut M.M., Elagin V.V., Sirotkina M.A., Medyanik I.A., Kravets L.Ya., Gladkova N.D. Multimodal optical coherence tomography for in vivo imaging of brain tissue structure and microvascular network at glioblastoma. In: Clinical and translational neurophotonics. Edited by Madsen S.J., Yang V.X.D. SPIE; 2017, https://doi. org/10.1117/12.2252286.

30. Matveev L.A., Zaitsev V.Y., Gelikonov G.V., Matveyev A.L., Moiseev A.A., Ksenofontov S.Y., Gelikonov V.M., Sirotkina M.A., Gladkova N.D., Demidov V., Vitkin A. Hybrid M-mode-like OCT imaging of three-dimensional microvasculature in vivo using reference-free processing of complex valued B-scans. Opt Lett 2015; 40(7): 1472-1475, https://doi.org/10.1364/ol.40.001472.
31. Matveev L.A., Karashtin D.A., Sovetsky A.A., Gubarkova E.V., Sirotkina M.A., Matveyev A.L., Shabanov D.V., Gelikonov G.V., Gelikonov V.M., Druzhkova I.N., Gladkova N.D., Vitkin A., Zagaynova E.V., Zaitsev V.Y. Quasistatic in-depth local strain relaxation/creep rate mapping using phase-sensitive optical coherence tomography. In: Optical coherence imaging techniques and imaging in scattering media II. Edited by Boppart S.A., Wojtkowski M., Oh W.-Y. SPIE; 2017, https://doi.org/10.1117/12.2284467.

32. Zaitsev V.Y., Matveyev A.L., Matveev L.A., Gelikonov G.V., Gubarkova E.V., Gladkova N.D., Vitkin A. Hybrid method of strain estimation in optical coherence elastography using combined sub-wavelength phase measurements and supra-pixel displacement tracking. J Biophotonics 2016; 9(5): 499-509, https://doi.org/10.1002/ jbio.201500203.

33. Zaitsev V.Y., Matveyev A.L., Matveev L.A., Gelikonov G.V., Sovetsky A.A., Vitkin A. Optimized phase gradient measurements and phase-amplitude interplay in optical coherence elastography. J Biomed Opt 2016; 21(11): 116005, https://doi.org/10.1117/1.jbo.21.11.116005.

34. Matveyev A.L., Matveev L.A., Sovetsky A.A., Gelikonov G.V., Moiseev A.A., Zaitsev V.Y. Vector method for strain estimation in phase-sensitive optical coherence elastography. Laser Phys Lett 2018; 15(6): 065603, https://doi. org/10.1088/1612-202x/aab5e9.

35. Sovetsky A.A., Matveyev A.L., Matveev L.A., Shabanov D.V., Zaitsev V.Y. Manually-operated compressional optical coherence elastography with effective aperiodic averaging: demonstrations for corneal and cartilaginous tissues. Laser Phys Lett 2018; 15(8): 085602, https://doi. org/10.1088/1612-202x/aac879.

36. Ralston T.S., Marks D.L., Carney P.S., Boppart S.A. Inverse scattering for optical coherence tomography. J Opt Soc Am A Opt Image Sci Vis 2006; 23(5): 1027-1037, https://doi. org/10.1364/fio.2006.fmi3.

37. Moiseev A.A., Gelikonov G.V., Ksenofontov S.Y., Shilyagin P.A., Terpelov D.A., Kasatkina I.V., Karashtin D.A., Sovetsky A.A., Gelikonov V.M. Digital refocusing in optical coherence tomography using finite impulse response filters. Laser Phys Lett 2018; 15(9): 095601, https://doi. org/10.1088/1612-202x/aaca60.

38. Moiseev A.A., Gelikonov G.V., Terpelov D.A., Shilyagin P.A., Gelikonov V.M. Digital refocusing for transverse resolution improvement in optical coherence tomography. Laser Phys Lett 2012; 9(11): 826-832, https://doi.org/10.7452/ lapl.201210102.

39. Rajadhyaksha M., Marghoob A., Rossi A., Halpern A.C., Nehal K.S. Reflectance confocal microscopy of skin in vivo: from bench to bedside. Lasers Surg Med 2017; 49(1): 7-19, https://doi.org/10.1002/lsm.2260. 